\title{
Identificación de las prioridades de conservación de la biodiversidad marina y costera en Costa Rica
}

\author{
Juan José Alvarado ${ }^{1,2,3}$, Bernal Herrera ${ }^{2}$, Lenin Corrales ${ }^{2}$, Jenny Asch ${ }^{4} \&$ Pía Paaby $^{5}$ \\ 1. Centro de Investigación en Ciencias del Mar y Limnología (CIMAR), Universidad de Costa Rica, San Pedro, 11501- \\ 2060 San José, Costa Rica; juanalva76@yahoo.com \\ 2. The Nature Conservancy, Región de Latinoamérica; bherrera@tnc.org, lcorrales@tnc.org \\ 3. Posgrado en Ciencias Marinas y Costeras, Universidad Autónoma de Baja California Sur, La Paz, México. \\ 4. Programa Marino-Costero, Sistema Nacional de Áreas de Conservación, Ministerio del Ambiente, Energía y \\ Telecomunicaciones, San José, Costa Rica; jasch@sinac.co.cr \\ 5. The Nature Conservancy (consultora); ppaaby@hotmail.com
}

Recibido 29-VI-2010. Corregido 10-XII-2010. Aceptado 11-I-2011.

\begin{abstract}
Identification of Marine and Coastal biodiversity conservation priorities in Costa Rica. Costa Rica is recognized as one of the most diverse countries in species and ecosystems, in their terrestrial realm as well as in the marine. Besides this relevance, the country presents a delay on conservation and management of marine and coastal biodiversity, with respect to terrestrial. For 2006, the marine protected surface was $5208.8 \mathrm{~km}^{2}$, with $331.5 \mathrm{~km}$ of coastline, in 20 protected areas. The country has made progress on the conservation priority sites identification for terrestrial and freshwater biodiversity, with few efforts on marine planning. This research presents the analysis and results of the gap identification process, for marine and coastal biodiversity conservation in the protected areas system of Costa Rica. The analysis was built with the spatial information available on the presence and distribution of coastal and marine biodiversity, the establishment of the conservation goals and a threat analysis over the ecological integrity of this biodiversity. The selection of high-priority sites was carried out using spatial optimization techniques and the superposition over the current shape of marine protected areas, in order to identify representation gaps. A total of $19076 \mathrm{~km}^{2}$ of conservation gaps were indentified, with $1323 \mathrm{~km}^{2}$ in the Caribbean and $17753 \mathrm{~km}^{2}$ in the Pacific. Recommendations are aimed at planning and strengthening the marine protected areas system, using the gaps identified as a framework. It is expected that the results of this study would be the scientific base needed for planning and sustainable use of marine biodiversity in the country. Rev. Biol. Trop. 59 (2): 829-842. Epub 2011 June 01.
\end{abstract}

Key words: conservation priority sites, conservation gaps, marine protected areas, marine conservation, conservation objects.

En el mundo se han identificado 34 regiones de alta diversidad de especies (Myers et al. 2000). Mesoamérica es una de ellas y resalta por varias condiciones: i) su gran cantidad de especies endémicas, ii) el papel que juega como corredor terrestre para las especies entre las dos masas continentales de Norte y Suramérica, y iii) ser el tercero en tamaño entre los centros de alta biodiversidad (Myers et al. 2000). Costa Rica, como parte de esta región, con sólo $51100 \mathrm{~km}^{2}$ de extensión en su territorio terrestre $(0.03 \%$ de la superficie mundial $)$, alberga alrededor de 90000 especies terrestres; aproximadamente el $4.5 \%$ de la biodiversidad global (Obando-Acuña 2002). Esta riqueza de especies se ve reforzada por su diversidad marina y costera. El país posee $568054 \mathrm{~km}^{2}$ de superficie marina total en la que se han identificado aproximadamente 6700 especies marinas (3.5\% de las especies marinas reportadas a nivel global), de las cuales 90 son endémicas (la mayoría en la Isla del Coco) (Wehrtmann 
\& Cortés 2009). La costa del Pacífico contiene la mayor cantidad de especies (4 700), mientras que el Caribe posee aproximadamente 2300. Asimismo, Costa Rica posee una gran diversidad de hábitats marinos, como arrecifes coralinos, manglares, fondos lodosos, zonas rocosas, playas, acantilados, praderas de pastos marinos, un fiordo tropical, áreas de surgencia, estuarios, un domo térmico, una fosa oceánica de más de $4000 \mathrm{~m}$ de profundidad, una dorsal oceánica, islas costeras, una isla oceánica (Cortés \& Werhtmann 2009) y ventilas hidrotermales (Bohrmann et al. 2002).

A pesar de esta riqueza de ambientes marinos, el país presenta un rezago en la conservación y manejo de la biodiversidad marina y costera, con respecto al esfuerzo realizado hacia la parte terrestre. Sin embargo, actualmente se realizan importantes esfuerzos en diferentes escalas, como la elaboración de la Estrategia Nacional para la Conservación y Manejo de los Recursos Marinos y Costeros (CIZEECR 2008). En el 2006, la superficie marina protegida era de $5208.8 \mathrm{~km}^{2}$, con $331.5 \mathrm{~km}$ de línea costera protegida, en 20 áreas silvestres protegidas (ASP); dos de ellas, exclusivamente marinas (los parque nacionales marinos Las Baulas y Ballena) (SINAC 2006). Sumado a esto, existen otras áreas protegidas que a pesar de no incluir una superficie marina, sí protegen la línea de costa. En este sentido, $611 \mathrm{~km}(48.7 \%)$ están bajo alguna categoría de manejo en el litoral Pacífico, mientras que en el Caribe, $114 \mathrm{~km}$ de costa $(53.8 \%)$ están protegidos. El área protegida costero-marina representa $0.9 \%$ de la superficie marina jurisdiccional total del país (SINAC 2006).

La sobreexplotación de recursos, la alteración física de hábitats, la contaminación, las invasiones biológicas y el cambio climático son reconocidas como las causas principales de pérdida de biodiversidad marina en el mundo (Breitburg \& Riedel 2005, Cortés \& Werhtmann 2009). Por esta razón, se hace necesario contar con información científica actualizada que permita el desarrollo de planes de conservación y uso sostenible de tal biodiversidad (Groves et al. 2002, Herrera \& Finegan 2008).
Esta información permitirá diseñar estrategias dirigidas a controlar o abatir las amenazas a la biodiversidad y a los servicios ecosistémicos (Herrera \& Finegan 2008).

Costa Rica presenta importantes avances en la determinación de sitios prioritarios para la conservación de la biodiversidad terrestre (Arias et al. 2008, www.gruas.go.cr) y aguas continentales (Paaby 2008). Sin embargo, hasta el momento existen pocos avances en la planificación para la conservación de la biodiversidad marina. En esta investigación, se presentan los vacíos en la representatividad de la biodiversidad marina y costera en el sistema de áreas protegidas de Costa Rica (referidos de aquí en adelante como "vacíos de conservación") y que deben ser conservados para asegurar una adecuada representatividad de los sistemas ecológicos, de las poblaciones de especies asociadas, su funcionalidad ecológica, así como la provisión continua de bienes y servicios ecosistémicos a las poblaciones humanas que de ellos dependen. De esta forma, el estudio complementa los esfuerzos realizados en planificación para la conservación del país.

\section{MATERIALES Y MÉTODOS}

El presente trabajo utilizó como punto de partida los resultados obtenidos para la determinación de sitios prioritarios de conservación a escala ecorregional desarrollado para Costa Rica, Panamá y Colombia (TNC 2008a, www. ecomeso.org); particularmente en lo referente a: (i) la información disponible sobre los objetos de conservación, (ii) el establecimiento de las metas de conservación y (iii) el análisis de las amenazas sobre la integridad ecológica de los objetos de conservación (OdC). Subsiguientes análisis utilizaron los pasos metodológicos para la identificación de los vacíos de conservación desarrollados por Dudley \& Parrish (2005).

Estratificación del área de estudio: El área de estudio corresponde a la Zona Económica Exclusiva (ZEE) de Costa Rica (Fig. 1). Entonces, se utilizaron como base del análisis las dos provincias biogeográficas incluidas en 


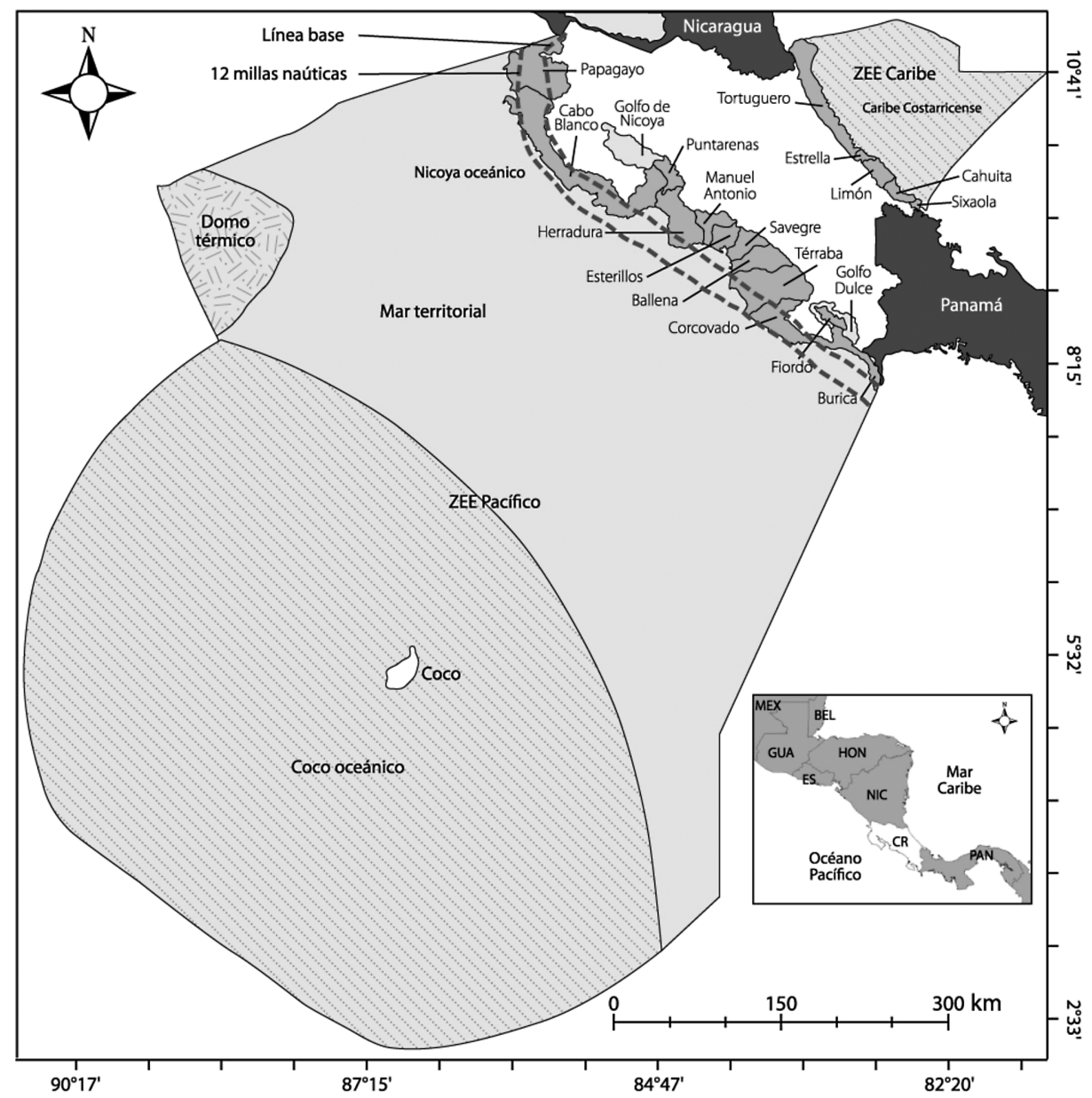

Fig. 1. Superficie marina patrimonial de Costa Rica y Unidades Ecológicas Marinas.

la zona económica exclusiva (ZEE) del país: Pacífico Tropical Oriental $\left(543842 \mathrm{~km}^{2}\right)$ y el Atlántico Tropical Noroccidental $\left(24212 \mathrm{~km}^{2}\right)$. Dentro de estas dos provincias, se han identificado dos ecorregiones en el océano Pacífico (Isla del Coco y Nicoya) y una en el Mar Caribe costarricense (Caribe Suroccidental) (Spalding et al. 2007). Para los esfuerzos nacionales de conservación de la biodiversidad, se consideró un alto grado de detalle que permitiera capturar una mayor variabilidad en la biodiversidad marina. Lo anterior debido a que ecológicamente, estas ecorregiones contienen sistemas relativamente homogéneos en la composición de especies y son consideradas como no apropiadas para la identificación de vacíos de conservación, como lo ha apuntado Jennings (2000) para otras regiones. Para esto, se identificaron unidades ecológicas marinas (UEM) (Fig. 1), a través de una estratificación de la ZEE, las cuales responden a la heterogeneidad ambiental generada por los gradientes en temperatura, profundidad, aportes de agua dulce y nutrimentos continentales, y morfología costera. Tales gradientes, en conjunto, son asociados con patrones diferentes en la composición de las comunidades marinas (Terán et al. 2006, TNC 2008a). Estas UEM aseguran que 
el proceso de planificación: se base en límites ecológicos, represente elementos desconocidos de la biodiversidad (e.g., posible variación genética en especies, dentro y entre comunidades en ecosistemas aparentemente muy similares), distribuya los sitios para dispersar los riesgos (e.g., evitar que eventos catastróficos locales afecten a toda la representación de un objeto de conservación particular), y genere unidades manejables para el análisis de datos (Beck 2003).

Identificación de los objetos de conservación marina: Los sistemas ecológicos se diferenciaron según la naturaleza del sustrato, ubicación en el fondo, la columna de agua, el litoral, la plataforma continental y la afectación de las mareas (TNC 2008a, b).
Con el fin de tomar en cuenta la heterogeneidad determinada por la profundidad y la morfología del fondo marino, se desarrolló un modelo batimétrico para la región Costa Rica-Panamá-Colombia el cual permite integrar en el análisis, los objetos de conservación definidos por esta heterogeneidad ambiental (TNC 2008a). Durante este esfuerzo, expertos de los diferentes países involucrados revisaron y validaron los criterios que generaron el modelo y los objetos de conservación viables y representativos para la región de análisis (TNC 2008a). Así, para el presente análisis de vacíos, en Costa Rica se trabajó con 26 sistemas ecológicos. El Cuadro 1 ofrece una descripción de los objetos de conservación (OdC) a nivel de sistemas ecológicos o de "filtro grueso" definidos para Costa Rica.

\section{CUADRO 1}

Caracterización de los sistemas ecológicos (objetos de conservación de filtro grueso) utilizados en el análisis de vacíos de la biodiversidad marina en Costa Rica

Playas de grano grueso

Playas de grano medio-fino

Planos intermareales de lodo (lodazales)

Playas rocosas

\section{Acantilados de roca dura}

Manglares / Manglares mixohalinos

Fondos de arena litoclástica en el sublitoral

Lodos litoclásticos en el sublitoral
Formaciones litorales de arena, parcialmente emergidas, con sedimentos predominantemente gruesos y en zonas expuestas al oleaje o de alta energía.

Formaciones litorales de arena, parcialmente emergidas, con sedimentos predominantemente finos y en zonas de poca a moderada energía o exposición al oleaje. Su pendiente es más suave que en las playas de arenas de grano grueso, por lo que suelen ser más anchas y estables.

Formaciones litorales de sedimentos finos que quedan emergidas durante la bajamar.

Formaciones litorales parcialmente emergidas compuestas por fragmentos líticos de dimensiones y origen variable, que van desde cantos rodados y gravas gruesas hasta bloques.

Formaciones litorales parcialmente emergidas, formadas por rocas masivas de consistencia dura (basaltos, granito, etc.), de pendiente fuerte a escarpada.

Zona boscosa meso y supralitoral que marca la transición entre los ámbitos marino y terrestre; forma una franja más o menos amplia en zonas costeras caracterizadas por planos aluviales influenciados por descargas de aguas dulces y sedimentos.

Fondos permanentemente sumergidos cubiertos por sedimentos de grano medio a grueso originados por fragmentación de rocas no carbonatadas, transportados y depositados sobre la plataforma continental o insular.

Fondos permanentemente sumergidos cubiertos por sedimentos de grano fino a muy fino originados por fragmentación de rocas no carbonatadas, transportados y depositados sobre la plataforma continental o insular. 
Lodos bioclásticos en el sublitoral

Arenas bioclásticas en el sublitoral

Fondos blandos batiales

Fondos duros batiales

Fondos blandos abisales

Fondos duros abisales

Fondos duros en el infralitoral

Fondos duros en el circalitoral

Praderas de pastos marinos

\section{Formaciones coralinas}

\section{Fosas anóxicas}

\section{Estuarios}

Lagunas costeras

\section{Sistema pelágico nerítico}

Fondos permanentemente sumergidos cubiertos por sedimentos de grano medio a grueso originados por fragmentación de esqueletos de plantas (algas calcáreas) y animales (foraminíferos, corales, moluscos, equinodermos) y que, por lo tanto, poseen un alto contenido de carbonatos.

Fondos permanentemente sumergidos cubiertos por sedimentos de grano fino a muy fino originados por fragmentación de esqueletos de plantas y animales y que por lo tanto poseen un alto contenido de carbonatos.

Fondos del talud continental o insular y de las áreas donde la profundidad de la columna de agua es de 200 a $2000 \mathrm{~m}$, cubiertos generalmente por sedimentos de grano fino a muy fino.

Son áreas de sustrato duro donde la pendiente del fondo es pronunciada a escarpada, generalmente asociados a zonas del talud continental o insular, laderas de montañas submarinas, cañones y grietas, y donde la profundidad de la columna de agua es de 200 a $2000 \mathrm{~m}$.

Fondos de las áreas más profundas (2 000 a $5000 \mathrm{~m})$ y de relieve relativamente plano del Pacífico Tropical Oriental, cubiertos supuestamente por limos orgánicos (cadáveres de organismos planctónicos principalmente).

Fondos de las áreas más profundas (2 000 a 5 000m) del Pacífico Tropical Oriental donde, por lo pronunciado de la pendiente, mayor a la del ángulo de reposo de la mayoría de los sedimentos marinos, es probable que sean de consistencia dura, formados probablemente por basaltos.

Lecho marino permanentemente sumergido hasta 60 metros de profundidad conformado por sustrato rocoso, generalmente formando altorrelieves (bajos rocosos).

Lecho marino permanentemente sumergido, entre 60 y $200 \mathrm{~m}$ de profundidad conformado por sustrato rocoso.

Lecho marino sedimentario, permanentemente sumergido, por lo general entre 0 y $10 \mathrm{~m}$ de profundidad, cubierto en gran parte por fanerógamas marinas (Ruppia maritima en el Pacífico y Thalassia testudinum y Syringodium filiformis en el Caribe) formando praderas.

Lecho marino permanentemente sumergido constituido en gran parte por colonias de corales hermatípicos ramificadas o masivas que determinan la existencia de una biota particular muy diversa.

Fondos submarinos situados por debajo de la capa de agua de mínimo oxígeno, cuyos fondos anóxicos son el hábitat de una biota muy particular (macrobacterias).

Cuerpos de agua semicerrados donde ocurre la mezcla de aguas marinas y dulces; columna de agua generalmente estratificada (cuña salina).

Cuerpos de aguas cerradas, de escasa profundidad, pero con una o más comunicaciones permanentes o esporádicas con el mar, generalmente con salinidad variable y elevada productividad planctónica.

Columna de agua marina localizada sobre la plataforma continental (hasta $200 \mathrm{~m}$ de profundidad) a lo largo de las costas continentales e insulares no oceánicas. 


\begin{abstract}
Áreas de surgencia
\end{abstract}
Islas e islotes

Montañas submarinas

Domo térmico
Columna de agua marina sujeta a movimientos ascendentes que producen afloramientos a la superficie de masas de agua de baja temperatura y nutrientes disueltos que desencadenan una alta productividad primaria y dan origen a una concentración de plancton, peces y mamíferos pelágicos.

Islotes rocosos e islas no deltaicas con tamaño inferior a 60 hectáreas. Este OdC se seleccionó como subrogado para obviar la falta de información sobre sitios de anidación de aves marinas.

Geoformas del fondo marino que generalmente revisten importancia para la biodiversidad. Se consideraron los sitios que cumplían con las siguientes condiciones: i) altura de más de $1000 \mathrm{~m}$ desde su base hasta su cima, ii) su cima o partes más elevadas alcanzan hasta la zona fótica $(0-200 \mathrm{~m})$, iii) la pendiente de sus laderas permite inferir la presencia de sustratos duros.

Centro de afloramiento oceánico permanente de 300-500km de diámetro, situado a unos 300km al oeste del Golfo de Papagayo en Costa Rica. Es considerada una zona de alta productividad primaria.

Para mayores detalles consultar TNC (2008a, b).

Para la selección de las especies, se definieron tres criterios para su incorporación en el ejercicio de priorización (TNC 2008b): 1) grado de amenaza global, según las categorías empleadas por la Comisión para la Supervivencia de Especies de la UICN (en peligro crítico, en peligro, vulnerable y casi amenazada; UICN 2001); 2) irremplazabilidad, que presta especial atención a las especies endémicas (con distribución restringida a una ecorregión o a una porción de ésta) y focales (con amplios requerimientos de espacio y función ecológica importante); 3) jurisdiccionalidad, la cual considera aquellas especies que tienen una importancia relevante en alguno de los países cuya jurisdicción marítima hace parte de las ecorregiones objeto de la evaluación (TNC 2008a).

Adicionalmente, se consideraron aquellas especies que, aunque no figuren en los listados de la UICN como globalmente amenazadas, están incluidas en los listados nacionales de especies amenazadas o en los Libros Rojos (www.iucnredlist.org). También, se incluyeron las especies migratorias cuya existencia depende de la calidad de los hábitats "de paso" dentro del área de evaluación. De esta forma, en total se definieron 25 OdC a nivel de especies o "filtro fino" para Costa Rica (Cuadro 2).

\section{Definición de las metas de conservación:}

Una meta de conservación se define como la cantidad (porcentaje o superficie) del OdC que debe ser conservado para mantener una población, comunidad o sistema ecológico en condiciones viables y que representen el amplio espectro de diversidad en una región (Groves 2003, Lourie \& Vincent 2004). La meta se representó mediante un valor numérico, y se estimó para cada OdC y para cada UEM.

El procedimiento para establecer las metas partió del supuesto de aspirar a conservar el $100 \%$ de las existencias en el momento de la evaluación de los OdC. Sin embargo, este porcentaje se redujo en función de factores de "penalización" (que varían entre 0.1 y 1.0), que se aplicaron de acuerdo al conocimiento y criterio de expertos sobre cada OdC. Los criterios utilizados para asignar tales valores fueron: 1) estado actual de salud de los OdC; 2) la vulnerabilidad de dichos elementos; 3 ) escala espacial (grande o pequeña), su distribución 
Objetos de conservación a nivel de unidades ecológicas marinas y especies (objetos de conservación de filtro fino) utilizados en el análisis de vacíos de conservación

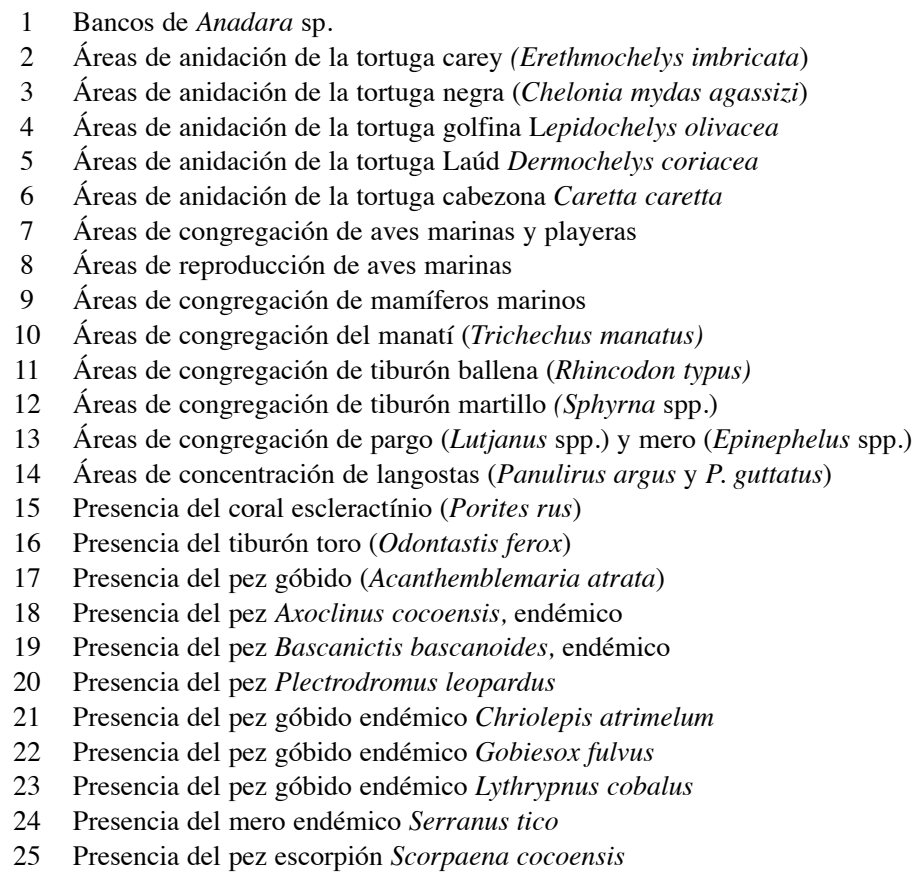

(amplia o limitada) y su arreglo (discreto o continuo) en la que ocurre el OdC y 4) la rareza y representación en la ocurrencia de tales elementos (TNC 2008a, b). Con la estimación de estos valores, las cuatro variables fueron promediadas y asignada de esta forma la meta a cada uno de los OdC.

Las metas de conservación estimadas, para cada uno de los objetos de conservación en cada una de las UEM varían entre 10 y $100 \%$. Para las montañas submarinas y las islas e islotes rocosos se estableció directamente una meta de $100 \%$ por su reducido tamaño, distribución dispersa e importancia. Por limitaciones de espacio, las metas de conservación no se presentan en el presente documento, pero se pueden consultar en los resultados de la evaluación ecorregional (TNC 2008a, b; http://www. ecomeso.org/).

Análisis de los factores de presión sobre la biodiversidad: El análisis de los factores de presión sobre la biodiversidad o amenazas consistió en dos fases. En la primera, se identificaron las actividades humanas que presentan presiones sobre los atributos ecológicos de los OdC. Para efectos de este estudio, se seleccionaron aquellas actividades humanas que tienen mayor relevancia, de acuerdo con la disponibilidad de información, la capacidad de cobertura espacial, y la posibilidad de cartografiar, y se agruparon según la forma en que pueden ser representadas espacialmente, a saber: 1) contaminación (doméstica e industrial); 2) infraestructura costera (incluyendo carreteras y líneas férreas); 3 ) navegación (rutas de navegación y operación portuaria, y dragados); y 4) extracción de recursos (pesca artesanal, pesca de recursos demersales, y pesca industrial de pelágicos). En una segunda fase, estas actividades fueron calificadas según cuatro componentes que caracterizan su potencial impacto: 1) probabilidad de que ocurran; 2) cobertura, 3) severidad y 4) permanencia o irreversibilidad. 
Estas valoraciones fueron realizadas mediante consulta a expertos para generar una superficie digital con la distribución de las magnitudes numéricas de los factores de presión que afectan la integridad y/o viabilidad de los OdC (TNC 2008a, b).

\section{Identificación de los vacíos de conserva-}

ción: La determinación de sitios prioritarios de conservación a nivel ecorregional fue realizado mediante la técnica de optimización espacial conocida como "recombinación simulada" (Possigham et al. 2000), la cual selecciona sitios compactos donde las amenazas sean mínimas, se presenta la mejor viabilidad de los OdC y se cumplen las metas de conservación establecidas (Possigham et al. 2000). Este procedimiento ha sido utilizado en estudios similares en diferentes regiones (i.e. Beck \& Odaya 2001, Airamé et al. 2003). Estudios realizados por Ban (2009) muestran que este procedimiento logra mantener una adecuada representación de los objetos de conservación incluidos en los datos analizados, lo cual es consecuente con el objetivo del análisis de vacíos tal y como se define en este artículo. Utilizando esta información como base del análisis, se procedió con la identificación de los vacíos y omisiones de conservación a nivel nacional. Los vacíos, son aquellos sitios que no están integrados a ninguna área protegida, mientras que las omisiones son aquellas superficies que colindan con alguna área protegida y deben ser anexadas a ésta con el objeto de incorporar ecosistemas importantes no incluidos, establecer una mejor conectividad. Para esto, se delineó la representación de cada uno de los OdC y se contrastó con el sistema actual de áreas protegidas informadas para el 2007. De esta manera, fue posible definir la proporción de cada OdC representada dentro y fuera de las áreas protegidas. Todo lo que queda por fuera de las áreas protegidas constituye entonces el vacío de conservación (Jennings 2000). Otra metodología que detalla aspectos de la escogencia de sitios prioritarios de conservación puede ser consultada en TNC (2008a, b; http://www.ecomeso.org/).

\section{RESULTADOS}

A nivel nacional, de los 51 OdC seleccionados, alrededor de $76 \%$ no se encuentra representado dentro de las áreas protegidas (Cuadro 3), siendo esta representatividad importante (ca.98\% del total) en el caso de los OdC que se expresan mediante unidades de área (e.g. manglares). Un 69\% de los OdC cuya expresión espacial es de presencias puntuales se encuentra bien representado en las áreas protegidas (Cuadro 3). De acuerdo con estos resultados, a nivel nacional hay cuatro OdC que no están representados en ninguna de las ASP (i.e. montañas submarinas, áreas de congregación del Rhincodon typus y de la ballena azul Balaenoptera musculus, y el domo térmico). Tanto en número de objetos de conservación como en el resto de los OdC evaluados, la propuesta de conservación es mayor en el Pacífico que en el Caribe (Cuadro 3).

El análisis dio como resultado la identificación de un total de 35 sitios prioritarios para la conservación de la biodiversidad marina y costera en Costa Rica (Fig. 2, Cuadro 4). La extensión total que abarcan estos sitios es de $21071 \mathrm{~km}^{2}$ contando el domo térmico, y 8 $781 \mathrm{~km}^{2}$ sin éste. Del total de sitios prioritarios, 30 sitios se ubican en el Pacífico (19 404km²), mientras que cinco se encuentran en el Caribe (1 666.70 $\mathrm{km}^{2}$ ). La extensión promedio de los sitios fue de $617.2 \mathrm{~km}^{2}$, siendo Punta El Indio el vacío más pequeño con $4.2 \mathrm{~km}^{2}$ y el Domo Térmico el más grande con $12289.9 \mathrm{~km}^{2}$ (Cuadro 4). La mayoría (10) de los sitios de importancia, poseen un tamaño entre 100 y $200 \mathrm{~km}^{2}$, seguido por siete entre 200 y $500 \mathrm{~km}^{2}$, cinco entre 10 y $20 \mathrm{~km}^{2}$, tres entre 20 y $50 \mathrm{~km}^{2}$, tres entre 50 y $100 \mathrm{~km}^{2}$, dos entre 500 y $1000 \mathrm{~km}^{2}$, dos entre 1000 y $1500 \mathrm{~km}^{2}$ y uno inferior a $10 \mathrm{~km}^{2}$, así como uno superior a $1500 \mathrm{~km}^{2}$.

A nivel de UEM, el estrato Domo Térmico posee el $58 \%$ del área total de los sitios de importancia, seguido por los UEM Coco Oceánico $(7.37 \%)$, Tortuguero $(4.11 \%)$ y Coco (3.65\%). El estrato Domo Térmico fue el que aportó la mayor cantidad de área, ya que todo el estrato fue seleccionado como un sitio de 
CUADRO 3

Resumen de la propuesta de conservación para Costa Rica, por territorio marino y por costa

Propuesta de conservación a nivel nacional

\begin{tabular}{|c|c|c|c|c|c|c|c|c|c|}
\hline & \multirow{2}{*}{ Total } & \multirow{2}{*}{$\mathrm{FF}$} & \multirow{2}{*}{ FG } & \multicolumn{3}{|c|}{ Dentro de áreas protegidas } & \multicolumn{3}{|c|}{ Vacíos (fuera de áreas protegidas) } \\
\hline & & & & Total & FF & FG & Total & $\mathrm{FF}$ & FG \\
\hline \# OdC & 51 & 25 & 26 & 47 & 23 & 24 & 39 & 14 & 25 \\
\hline Extensión lineal (km) & 2298 & 806 & 4926 & 838 & 336 & 502 & 1460 & 469 & 991 \\
\hline Localidades puntuales & 29 & 25 & 4 & 21 & 19 & 2 & 9 & 7 & 2 \\
\hline Área $\left(\mathrm{km}^{2}\right)$ & 639188 & 44442 & 594746 & 8129 & 2270 & 5859 & 631059 & 42171 & 588888 \\
\hline \multicolumn{10}{|c|}{ Propuesta de conservación Océano Pacífico } \\
\hline \# OdC & 46 & 22 & 24 & 43 & 21 & 22 & 28 & 6 & 22 \\
\hline Extensión lineal (km) & 1691 & 426 & 1265 & 546 & 154 & 392 & 1145 & 272 & 873 \\
\hline Localidades puntuales & 27 & 23 & 4 & 23 & 19 & 4 & 4 & 4 & 0 \\
\hline Área $\left(\mathrm{km}^{2}\right)$ & 617637 & 44351 & 573286 & 7240 & 2197 & 5043 & 610397 & 42154 & 568243 \\
\hline \multicolumn{10}{|c|}{ Propuesta de conservación Mar Caribe } \\
\hline \# OdC & 26 & 10 & 16 & 22 & 9 & 13 & 23 & 7 & 16 \\
\hline Extensión lineal (km) & 607 & 380 & 227 & 292 & 182 & 110 & 315 & 198 & 117 \\
\hline Localidades puntuales & $2\left(+5.7 \mathrm{~km}^{2}\right)$ & 2 & $5.7 \mathrm{~km}^{2}$ & $2\left(+4.2 \mathrm{~km}^{2}\right)$ & 2 & $4.2 \mathrm{~km}^{2}$ & $0\left(+1.5 \mathrm{~km}^{2}\right)$ & 0 & $1.5 \mathrm{~km}^{2}$ \\
\hline Área $\left(\mathrm{km}^{2}\right)$ & 21551 & 91 & 21460 & 889 & 73 & 816 & 20662 & 18 & 20644 \\
\hline
\end{tabular}

Para efecto de los análisis los objetos de conservación (OdC) pueden expresarse en términos de área (e.g. manglares), líneas (e.g. playas) o presencias puntuales (e.g. sitios de anidación). OdC: Objeto de conservación; FG: Filtro Grueso; FF: Filtro Fino.

CUADRO 4

Propuesta de sitios para la conservación de la biodiversidad marina

\begin{tabular}{clccc} 
No. & \multicolumn{1}{c}{ Sitio } & Área $\left(\mathrm{km}^{2}\right)$ & Área dentro de ASP $\left(\mathrm{km}^{2}\right)$ & Vacío de conservación $\left(\mathrm{km}^{2}\right)$ \\
1 & Descartes & 46 & 0 & 46 \\
2 & Bahía Santa Elena & 16 & 5 & 11 \\
3 & Punta Santa Elena & 193 & 76 & 117 \\
4 & Golfo de Papagayo & 179 & 54 & 125 \\
5 & Punta Gorda - Punta Pargos & 247 & 23 & 224 \\
6 & Ostional & 86 & 86 & 0 \\
7 & Punta El Indio & 4 & 0 & 4 \\
8 & Cabo Blanco & 162 & 23 & 139 \\
9 & Punta Tambor & 12 & 0 & 20 \\
10 & Curú - IslasTortugas & 21 & 1 & 154 \\
11 & Negritos - San Lucas & 164 & 10 & 66 \\
12 & Caballo - Venado & 66 & 0 & 271 \\
13 & Chira - Tempisque & 329 & 58 & 28 \\
14 & Estero Culebra & 28 & 0 & 53 \\
15 & Aranjuez & 53 & 0 & 89 \\
16 & Caldera - Tárcoles & 89 & 0 & 14 \\
17 & Herradura & 14 & 0 & 325 \\
18 & Punta Judas & 325 & 0 & 137 \\
19 & Damas - Savegre & 185 & 48 &
\end{tabular}


CUADRO 4 (Continuación)

Propuesta de sitios para la conservación de la biodiversidad marina

\begin{tabular}{clccc} 
No. & \multicolumn{1}{c}{ Sitio } & Área $\left(\mathrm{km}^{2}\right)$ & Área dentro de ASP $\left(\mathrm{km}^{2}\right)$ & Vacío de conservación $\left(\mathrm{km}^{2}\right)$ \\
20 & Dominical - Sierpe & 478 & 33 & 445 \\
21 & Plataforma de Coronado & 37 & 0 & 37 \\
22 & Isla del Caño & 168 & 58 & 110 \\
23 & Plataforma de Osa & 131 & 0 & 131 \\
24 & Corcovado & 232 & 113 & 119 \\
25 & Montañas submarinas de Osa & 157 & 0 & 157 \\
26 & Golfo Dulce & 1153 & 68 & 1086 \\
27 & Punta Burica & 224 & 0 & 224 \\
28 & Montañas submarinas de Coco & 1311 & 0 & 1311 \\
29 & Isla del Coco & 1002 & 995 & 7 \\
30 & Domo térmico & 12290 & 0 & 12290 \\
31 & Barra del Colorado & 967 & 7 & 960 \\
32 & Canales de Tortuguero & 147 & 56 & 91 \\
33 & Isla Uvita & 17 & 0 & 17 \\
34 & Cahuita & 373 & 233 & 141 \\
35 & Gandoca & 163 & 48 & 115 \\
Total & & 21071 & 1995 & 19076 \\
Caribe & & 1667 & 344 & 1323 \\
Pacífico & 19404 & 1651 & 17753
\end{tabular}

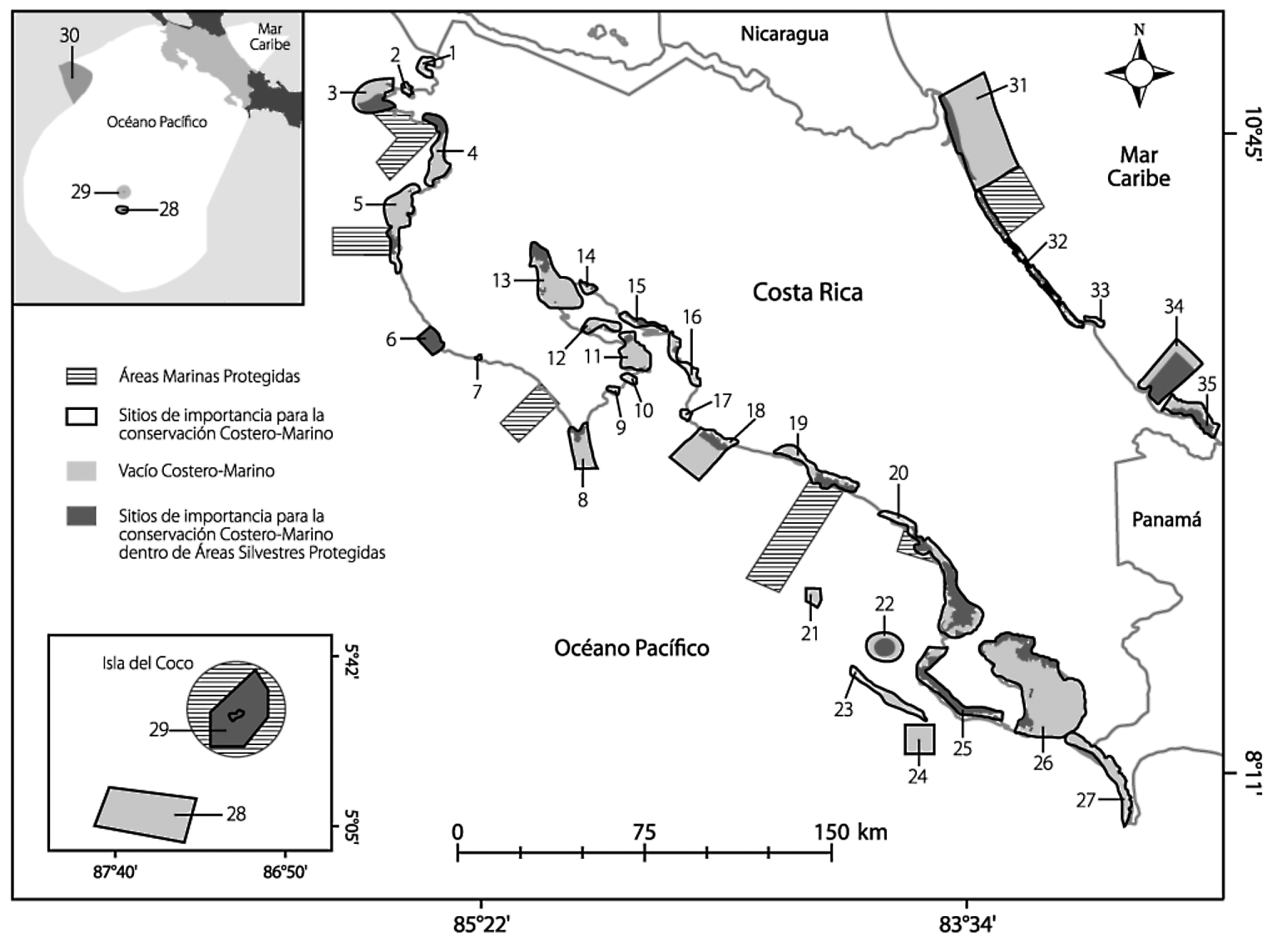

Fig. 2. Vacíos de conservación de la biodiversidad marina en Costa Rica. 
importancia (Fig. 2). Es necesario recalcar que el domo térmico de Costa Rica es un evento oceanográfico dinámico que crece y desaparece de acuerdo con las condiciones oceanográficas y atmosféricas de la región (Fiedler 2002), y que el estrato considerado en este estudio representa la ubicación promedio que posee este fenómeno oceanográfico de acuerdo a Fiedler (2002). El estrato Estrella fue el que aportó la menor cantidad porcentual al total de sitios de importancia en el Caribe. Los UEM Limón y Estrella son los que aportaron menos con $0.1 \%$ cada uno.

En total se hallaron $19076 \mathrm{~km}^{2}$ como vacíos de conservación (sitios prioritarios por fuera de alguna categoría de manejo), lo que corresponde al $88.1 \%$ de los sitios de importancia. En este caso, el tamaño promedio de los vacíos fue de $543.7 \mathrm{~km}^{2}$, y al igual que con los sitios de importancia, el vacío más grande corresponde al Domo Térmico y el más pequeño a Punta El Indio. El estrato que presentó el mayor porcentaje del área total de los vacíos fue de nuevo el Domo Térmico. Este vacío corresponde en su totalidad al sitio y al estrato con el mismo nombre.

El ambiente oceánico es el que aportó el mayor porcentaje de área a los vacíos de conservación marino-costera con un $76.6 \%$, mientras que los aportes que hacen los ambientes de línea de costa y nerítico son similares, ambos con un $9 \%$. Así mismo, existe un 5.3\% encontrado fuera de estos ambientes y corresponde a las secciones terrestres de los sitios. De la misma manera que sucedió con los sitios de importancia, al analizar el área que cubre cada sitio dentro del total de cada ambiente, los números se invierten. En este caso, es en el ambiente costero donde se encontró el mayor porcentaje, ya que los vacíos abarcaron un 40.4\% del total de ambiente de línea de costa que posee Costa Rica, mientras que en el caso de los otros ambientes son porcentajes bajos.

\section{DISCUSIÓN}

Es importante recalcar que a pesar de que la mayoría de los vacíos de conservación se encuentran en la parte oceánica, es muy poco lo que representa de este ambiente para todo el país $(2.6 \%)$. Por otro lado, del total de vacíos de conservación identificados, $9.1 \%$ están en la línea de costa, y a diferencia del ambiente anterior, es en éste donde se evidencia la mayor cantidad de vacíos (40.4\%). Esto refleja dos hechos: 1) que los esfuerzos de investigación y conservación en Costa Rica se han centrado sobre todo en la parte costera del país, y 2) que la investigación se ha centrado en la costa Pacífica donde se encuentra el $76.1 \%$ del total de sitios de importancia para el ambiente de línea de costa, el $79.2 \%$ para el ambiente nerítico y el $96.9 \%$ para el ambiente oceánico.

El mayor número de sitios de importancia y de vacíos aparecen en el Área de Conservación Osa (Pacífico suroeste del país) y en el Área de Conservación Marina Isla del Coco, donde se han realizado considerables esfuerzos de investigación en los últimos años. Es importante recalcar el hecho de que prácticamente no hayan aparecido sitios de importancia para el Área de Conservación Tempisque (Pacífico Norte) responde a la falta de conocimiento general de la parte marina en esa zona del país, y se debe a las fuertes presiones que en ella se encuentran. El análisis refleja el esfuerzo de investigación por grupos y zonas en temas marinos y costeros en Costa Rica como los correspondientes a la Península de Santa Elena, Bahía Culebra, Osa y el Caribe sur, y entre los grupos, a los de cetáceos, las tortugas, arrecifes coralinos y manglares.

En este sentido, este análisis de vacíos de conservación no sólo refleja hacia donde deben de encaminarse los esfuerzos de conservación, sino también de investigación. El estudio sólo contempla, en el caso de organismos pelágicos móviles, a los cetáceos y tortugas, zonas de agregación para eventos reproductivos como son las playas o zonas de alumbramiento. Sin embargo, no contempla rutas migratorias críticas para estas especies u otros grupos de pelágicos que no fueron incluidos en este análisis (e.g. atunes, pez Vela), debido a la falta de información espacial requerida para este tipo de análisis (Dudley \& Parrish 2005). 
Así mismo, a pesar de conocer de que existen fuentes hidrotermales y sumideros fríos asociados a la plataforma continental (Bohrmann et al. 2002), la información está dispersa y poco accesible para los tomadores de decisión.

El área total de los OdC incluidos en la propuesta de conservación (639 188km²) sobrepasa la superficie de mar patrimonial (568 $054 \mathrm{~km}^{2}$ ), debido a que muchos de los OdC se traslapan. Como resultado de la unión de estas propuestas, se obtuvo entonces una iniciativa para los sitios prioritarios de conservación. La propuesta de conservación con todos los OdC cubre un área que alcanza $21071 \mathrm{~km}^{2}$; de los cuales $1995 \mathrm{~km}^{2}(9.5 \%)$ están dentro de las ASP actuales del país y $19076 \mathrm{~km}^{2}(90.5 \%)$ por fuera, y constituyen lo que se define como el vacío de conservación (Cuadro 4). Es claro que una cobertura menor al $1 \%$ del territorio marino nacional dentro de ASP no refleja la importancia de la biodiversidad marina presente en Costa Rica, ni es consecuente con el actual estado de conservación de estos recursos. La propuesta de conservación (i.e. el vacío de 19 $076 \mathrm{~km}^{2}$ ) deberá plasmarse mediante el diseño e implementación de estrategias de conservación para $1323 \mathrm{~km}^{2}$ en el Caribe y $17753 \mathrm{~km}^{2}$ en el Pacífico.

Como parte de la propuesta nacional, se consideró crítica la inclusión del Refugio Nacional de Vida Silvestre Ostional dentro de los sitios de importancia, puesto que no fue identificado en el proceso de escogencia de sitios prioritarios realizada utilizando "recombinación simulada". En Costa Rica hay dos sitios de arribada: playa Nancite y playa Ostional; sin embargo, esta última es la más importante, con aproximadamente diez arribadas de la tortuga lora (Lepidochelys olivacea) por año y estimados de 30000 a 135000 tortugas en cada arribada (Russell et al. 1999).

Dado que, el porcentaje de superficie marina nacional incluido dentro del sistema de áreas protegidas no alcanza el $1 \%$ de las aguas jurisdiccionales del país, y que este estudio ha determinado la existencia de importantes vacíos de conservación marina, es imperativo ampliar y fortalecer el sistema de áreas marinas protegidas e implementar el uso de otras herramientas de manejo y conservación de recursos que permitan el cumplimiento de las metas de conservación. Lo anterior se lograría a través de la asignación de categorías de manejo más permisivas, desde el punto de vista de intervención humana, como los son las Reservas Marinas y Áreas Marinas de Manejo, de acuerdo a la normativa nacional. Esto deberá analizarse caso por caso y teniendo en cuenta las condiciones socioeconómicas, culturales e institucionales locales y regionales de cada vacío identificado. Los resultados de los vacíos en la conservación de la biodiversidad terrestres y aguas continentales deberán integrarse en este proceso de implementación con el fin de garantizar acciones lógicas y acordes con la conectividad intrínseca de los sistemas ecológicos.

Más allá de las ASP con superficie marina, el Sistema Nacional de Áreas de Conservación de Costa Rica (SINAC) no considera el manejo y conservación de los recursos marinos nacionales dentro de la zona económica exclusiva. Se recomienda impulsar el desarrollo e implementación de criterios de zonificación marina que faciliten el manejo y conservación integral de estos recursos en la ZEE del país. Vale la pena resaltar el problema administrativo para la gestión de los recursos naturales que enfrenta Costa Rica, ya que la división del territorio dentro de las once áreas de conservación no toma en cuenta la superficie marina, la cual representa un área con un tamaño más de diez veces superior al territorio terrestre del país. Es necesario entonces que futuros esfuerzos gubernamentales resuelvan este vacío para asegurar el respaldo institucional requerido para la implementación de los resultados del presente diagnóstico. Así mismo, la administración de los recursos marino-costeros está dispersa en varias instancias del Gobierno con poca coordinación entre ellas, y las cuales entran en conflicto en varias ocasiones, lo que dificulta una correcta administración.

Por otra parte, al comparar distintas zonas y disciplinas, resulta claro que la disponibilidad de información científica sobre ambientes marinos, tanto a escala regional como nacional, 
es insuficiente y desigual. Se recomienda promover la investigación científica marina en general, tanto en zonas costeras como oceánicas, en temas como: 1) pesquerías (biología pesquera, mejoramiento de bases de datos y estadísticas pesqueras, distribución de organismos pelágicos, centros de agregación de desove de peces); 2) oceanografía (física, química, geológica y biológica); 3) conectividad física y biológica de ecosistemas marinos y áreas marinas protegidas, a escalas local y regional; e 4) impacto de las principales amenazas (contaminación, sobreexplotación, cambio climático) sobre el estado de los ecosistemas marinos.

\section{AGRADECIMIENTOS}

El presente trabajo se realizó gracias a la colaboración de múltiples instituciones y expertos nacionales e internacionales, quienes desinteresadamente brindaron mucha de la información necesaria para realizar este trabajo. Un especial agradecimiento a F. Sánchez, M. Coto, L. Monge, P. Imbach, L. Guillermo Molina, J.M. Díaz, M. Quesada, J. Cortés y S. Echeverría quienes brindaron su apoyo en diferentes fases de la investigación y redacción del trabajo. El financiamiento fue posible gracias al patrocinio de The Nature Conservancy, Conservation International y el Sistema Nacional de Áreas de Conservación.

\section{RESUMEN}

Costa Rica es un país reconocido por su alta diversidad de especies y ecosistemas, tanto en sus ambientes terrestres como marinos. A pesar de esta importancia, presenta un rezago en la conservación y manejo de la biodiversidad marina y costera, con respecto a la terrestre. Para el año 2006, la superficie marina protegida era de $5208.8 \mathrm{~km}^{2}$ y $331.5 \mathrm{~km}$ de línea costera, en 20 áreas silvestres protegidas. El país ha logrado importantes avances en la selección de sitios prioritarios para la conservación de la biodiversidad terrestre y de aguas continentales, aunque pocos esfuerzos se han realizado hasta el momento en la planificación marina. En este trabajo se muestra el análisis y resultados de un proceso de identificación de vacíos en la representatividad de la biodiversidad marina y costera en el sistema de áreas protegidas de Costa Rica. El estudio se construyó a partir de la información espacial disponible sobre la presencia y distribución de la biodiversidad marina y costera, el establecimiento de metas de conservación y el análisis de amenazas sobre la integridad ecológica de la biodiversidad. Posteriormente, se llevó a cabo la selección de sitios prioritarios mediante técnicas de optimización espacial, y la sobreposición con la actual capa de áreas marinas protegidas, para finalmente identificar los vacíos en la representatividad. En total, se identificaron 19 $076 \mathrm{~km}^{2}$ de vacíos de conservación, $1323 \mathrm{~km}^{2}$ en el Caribe y $17753 \mathrm{~km}^{2}$ en el Pacífico. Se plantean recomendaciones dirigidas hacia la ampliación y fortalecimiento del sistema de áreas marinas protegidas del país, en el cual se usan los vacíos identificados como marco de referencia. Se espera que los resultados presentes sean parte de la base científica necesaria para la planificación de la conservación y uso sostenible de la biodiversidad marina en el país.

Palabras claves: sitios prioritarios de conservación, vacíos y omisiones en conservación, áreas marinas protegidas, conservación marina, objetos de conservación.

\section{REFERENCIAS}

Airamé, S., J.E. Dugan, K.D. Lafferty, H. Leslie, D.A. McArdle \& R.R. Warner. 2003. Applying ecological criteria to Marine Reserve design: A case study from the California Channel Islands. Ecol. Appl. 13: 170-184.

Arias, E., O. Chacón, G. Induni, B. Herrera, H. Acevedo, L. Corrales, J.B. Barborak, M. Coto, J. Cubero \& P. Paaby. 2008. Identificación de vacíos en la representatividad de ecosistemas terrestres en el Sistema Nacional de Áreas Protegidas de Costa Rica. Rec. Nat. Amb. 54: 21-27.

Ban, N. 2009. Minimum data requirements for designing a set of marine protected areas, using commonly available abiotic and biotic datasets. Biodiversity \& Conservation 18: 1829-1845.

Beck, M. 2003. The sea around: conservation planning in marine ecoregions, p. 319-365. In C.R. Groves (ed.). Drafting a Conservation blue-print: a practitioner's guide to planning for Biodiversity. Island, Washington D.C., EEUU.

Beck, M. \& M. Odaya. 2001. Ecoregional planning in marine environments: Identifying priority sites for conservation in the northern Gulf of Mexico. Aquatic Conservation 11: 235-242.

Bohrmann, G., K. Heeschen, C. Jung, W. Weinrebe, B. Baranov, B. Cailleau, R. Heath, V. Huhnerbach, M. Hort, D. Masson \& I. Trummer. 2002. Widespread fluid expulsion along the seafloor of the Costa Rica convergent margin. Terra Nova 14: 69-79. 
Breitburg, D.L. \& G.F. Riedel. 2005. Multiple stressors in marine systems, p. 167-182. In E. Norse \& L. Crowder (eds.). Marine conservation biology: the science of maintaining the sea's biodiversity. Island, Washington D.C., EEUU.

CIZEE-CR (Comisión Interinstitucional de la Zona Económica Exclusiva de Costa Rica). 2008. Estrategia nacional para la gestión integral de los recursos marinos y costeros de Costa Rica. Comisión Interinstitucional de la Zona Económica Exclusiva de Costa Rica, San José, Costa Rica.

Cortés, J. \& I.S. Wehrtmann. 2009. Diversity of marine habitats of the Caribbean and Pacific of Costa Rica, p. 1-45. In I.S. Wehrtmann \& J. Cortés (eds.). Marine Biodiversity of Costa Rica, Central America. Monogr. Biol. 86, Springer, Berlin.

Dudley, N. \& J. Parrish. 2005. Cubriendo los vacíos: la creación de áreas protegidas ecológicamente representativas. The Nature Conservancy, Mérida, Mexico.

Fiedler, P.C. 2002. The annual cycle and biological effects of the Costa Rica Dome. Deep-Sea Research I 49: 321-338.

Groves, C.R. 2003. Drafting a conservation blueprint: a practitioners guide to planning for biodiversity. The Nature Conservancy, Washington D.C., EEUU.

Groves, C.R., D.B. Jensen, L.L. Valutis, K.H. Redford, M.L. Shaffer, J.M. Scott, J.V. Baumgartner, J.V. Higgins, M.W. Beck \& M.G. Anderson. 2002. Planning for biodiversity conservation: putting conservation science into practice. BioScience 52: 499-512.

Herrera, B. \& B. Finegan. 2008. La planificación sistemática como instrumento para la conservación de la biodiversidad. Experiencias recientes y desafíos en Costa Rica. Rec. Nat. Amb. 54: 4-13.

Jennings, M.D. 2000. Gap analysis: concepts, methods, and recent results. Landsc. Ecol. 15: 5-20.

Lourie, S.A. \& S.C.J. Vincent. 2004. Using biogeography to help set priorities in marine conservation. Conserv. Biol. 18: 1004-1020.

Myers, N., R.A. Mittermeier, C.G. Mittermeier, G.A.B. da Fonseca \& J. Kent 2000. Biodiversity hotspots for conservation priorities. Nature 403: 853-858.

Obando-Acuña, V. 2002. Biodiversidad en Costa Rica: Estado del conocimiento y gestión. INBio, Santo Domingo de Heredia, Costa Rica.

Paaby, P. 2008. Vacíos en los esfuerzos de conservación de la biodiversidad de aguas continentales en Costa Rica. Rec. Nat. Amb. 54: 28-36.
Possigham, H.P., I.R. Ball \& S. Andelman. 2000. Mathematical methods for identifying representative reserves networks, p. 291-305. In S. Ferson \& M. Burgman (eds.). Quantitative Methods for Conservation Biology. Springer-Verlag, New York, EEUU.

Russell, A., S. Mehta \& R. Arauz. 1999. Analysis of synchronized mass nesting activity by Olive Ridley Sea Turtles (Lepidochelys olivacea) in the Ostional Wildlife Refuge, Guanacaste. Oral presentation. $19^{\text {th }}$ Ann. Sea Turtle Symp., South Padre Island, Texas, EEUU.

SINAC (Sistema Nacional de Áreas de Conservación). 2006. El sistema de áreas silvestres protegidas. Informe Nacional. II Congreso Mesoamericano de Áreas Protegidas, San José.

Spalding, M.D., H.E. Fox, G.R. Allen, N. Davidson, Z.A. Ferdaña, M. Finlayson, B.S. Halpern, M.A. Jorge, A. Lombana, S.A. Lourie, K.D. Martin, E. McManus, J. Molnar, C.A. Recchia \& J. Robertson. 2007. Marine ecoregions of the world: a bioregionalization of coastal and shelf areas. Bioscience 57: 573-583.

Terán, M.C., K. Clark, C. Suárez, F. Campos, J. Denkinger, D. Ruiz \& P. Jiménez. 2006. Análisis de vacíos e identificación de áreas prioritarias para la conservación de la biodiversidad marino-costera en el Ecuador continental. Resumen Ejecutivo. Ministerio del Ambiente. Quito, Ecuador.

TNC (The Nature Conservancy). 2008a. Evaluación de ecorregiones marinas en Mesoamérica; sitios prioritarios para la conservación en las ecorregiones Bahía de Panamá, Isla del Coco y Nicoya del Pacífico Tropical Oriental y en el Caribe Suroccidental de Costa Rica y Panamá. The Nature Conservancy, Programa de Ciencias Regional, Región de Mesoamérica y el Caribe, San José, Costa Rica.

TNC (The Nature Conservancy). 2008b. Proceso metodológico para la evaluación ecorregional marina en Mesoamérica: ecorregiones Bahía de Panamá, Isla del Coco y Nicoya del Pacífico Tropical Oriental y en el Caribe Suroccidental de Costa Rica y Panamá. The Nature Conservancy, Programa de Ciencias Regional, Región de Mesoamérica y el Caribe, San José, Costa Rica.

UICN (Union Internacional para la Conservación de la Naturaleza). 2001. Categorías y criterios de la Lista Roja de la UICN: Versión 3.1. Comisión de Supervivencia de Especies de la UICN. UICN, Gland, Suiza y Cambridge, Reino Unido.

Wehrtmann, I.S. \& J. Cortés (eds.). 2009. Marine Biodiversity of Costa Rica, Central America. Springer, Berlin. 